Archives

$17 \mid 1996$

Hommage à Bernard Lepetit

\title{
Pour une problématique de la recherche historique sur l'habitat. La maison : espace social
}

\section{Peter Granasztói}

\section{(2) OpenEdition}

Journals

\section{Édition électronique}

URL : http://journals.openedition.org/ccrh/2614

DOI : $10.4000 /$ ccrh. 2614

ISSN : $1760-7906$

Éditeur

Centre de recherches historiques - EHESS

Édition imprimée

Date de publication : 4 octobre 1996

ISSN : 0990-9141

Référence électronique

Peter Granasztói, «Pour une problématique de la recherche historique sur l'habitat. La maison : espace social », Les Cahiers du Centre de Recherches Historiques [En ligne], 17| 1996, mis en ligne le 27 février 2009, consulté le 10 décembre 2020. URL : http://journals.openedition.org/ccrh/2614 ; DOI https://doi.org/10.4000/ccrh.2614

Ce document a été généré automatiquement le 10 décembre 2020.

Article L.111-1 du Code de la propriété intellectuelle. 


\title{
Pour une problématique de la recherche historique sur l'habitat. La maison : espace social
}

\author{
Peter Granasztói
}

Quand on réfléchit sur l'habitation,

[...] il faut en voir l'élément très ancien, éternel peut-être : le reflet du séjour de

l'être humain dans le sein maternel'2.

2 Le chercheur qui s'intéresse à l'histoire de l'habitat ne doit pas étudier seulement la morphologie des maisons ou la typologie des meubles. Il doit plutôt essayer de découvrir quels sont les gestes de nos ancêtres qui se cachent derrière la forme de la maison et derrière les objets intérieurs. Pourquoi les hommes habitaient-ils ainsi et quels sont les phénomènes qui expliquent la diversité de l'habitat dans le temps et dans l'espace ? Nous sommes confrontés à ces questions en permanence.

3 Excepté quelques études spécialisées d'ethnologues, d'anthropologues, d'architectes, de sociologues et d'historiens, l'habitat fait rarement l'objet d'une véritable «histoire de l'habitat ». Cette énumération prouve la richesse de ce sujet et l'intérêt d'élaborer une approche plus complexe, une approche interdisciplinaire.

4 Rares sont les approches qui intègrent plusieurs types d'indicateurs (physiques, sociaux, culturels, économiques). Pourtant, plusieurs études, ces vingt dernières années, insistent sur la complexité de ce que recouvre l'habitat. Elles mettent de ce fait le doigt sur les lacunes des études précédentes qui n'ont analysé que la forme ou les caractéristiques physiques de la maison et son aménagement intérieur 3 .

5 Les nouvelles approches anthropologiques, ethnologiques et historiques étudient l'habitat comme forme d'activité socioculturelle et essaient

[...] de saisir l'habitat et l'habitation comme des objets totaux, comme le lieu d'intersection de multiples logiques, logique écologique, logique technique, logique économique ; logique sociale ou logique symbolique 4 .

6 La réalisation - dans le cadre d'une étude historique - d'un tel objectif semble difficile. Tandis que l'ethnologue observe directement la vie dans la maison, le comportement des 
habitants, à l'écoute de leurs désirs, l'historien, lui, est plongé dans les archives... Et il existe peu de sources pour retrouver les gestes symboliques ou « les expériences psychosubjectives des habitants » du passé5. On ne retrouve que la trace (matériaux, objets...) de l'existence de l'homme dans la maison: « celle-ci porte l'empreinte de celui qui l'occupe » ${ }^{6}$. Ces empreintes ont été conservées presque parfaitement par les inventaires après décès, par les plans des maisons, etc. Ainsi le point de départ de l'historien est de l'ordre des caractéristiques formelles ou physiques, donc de l'ordre de la culture matérielle. Cette dernière est l'indicateur premier de tous les phénomènes qui l'intéressent.

7 L'historien

[...] doit analyser les manières d'appropriation de l'espace, les diverses façons d'arranger l'extérieur de la maison et d'aménager l'intérieur, car chaque forme de vie en commun se reflète dans un aménagement précis de l'espace ${ }^{7}$.

8 Le but principal de l'historien est de retrouver les « facteurs » qui expliquent la diversité des formes de l'habitat. Étant donné que l'individu se trouve devant un choix quand il construit ou loue une maison, et qu'il y met des objets, la forme de la maison est le résultat du choix entre les possibilités existantes. Ce choix dépend donc toujours de facteurs qui sont différents selon les époques et les lieux, selon les individus et les groupes sociaux.

9 Les facteurs physiques, comme les matériaux à disposition, le climat, le niveau des techniques, sont certes déterminants dans ces choix et ne doivent pas être négligés. Mais les contraintes physiques ne sont pas exclusives. Selon Amos Rapoport ${ }^{8}$, l'influence des facteurs socioculturels est beaucoup plus importante et même primordiale. La différence des situations familiale, sociale, et de fortune-c'est-à-dire les différentes valeurs, normes, comportements symboliques ou modes de vie-explique véritablement la diversité des formes que prend la maison. Ces contraintes socioculturelles sont encore plus importantes dans une communauté traditionnelle comme la Grande Plaine, en Hongrie ${ }^{9}$.

10 Les exemples donnés par l'ethnologue hongroise Klara K. Csilléry prouvent également l'influence des facteurs socioculturels sur l'aménagement intérieur. Choisir et mettre un fauteuil dans une chambre avait une signification sociale profonde à Dunapataj, village hongrois, au début du xx $x^{e}$ siècle ; l'utilisation de ce fauteuil, qui exigeait un certain rang social (être propriétaire d'au moins 15 à 20 acres de terre ${ }^{10}$ ), était attentivement surveillé par la communauté.

11 Une famille pauvre (de journaliers) s'était procurée un fauteuil dans les années 1960, mais elle ne s'en servait pas et l'avait placé dans un coin de la chambre. Le chef de famille, conscient de sa situation sociale, ne voulait pas le mettre à côté de la table, et quand, à la demande de l'ethnologue, il s'y est assis en imitant les grands fermiers, il ne put y prendre position confortablement ${ }^{11}$.

12 Cet exemple montre que l'habitat peut abriter différents types de caractéristiques ou de comportements "psycho-subjectifs». Mais il faut aussi, immédiatement, tempérer ces propos en soulignant l'influence de la volonté propre de l'individu :

Chaque individu agit en fonction d'une situation qui lui est propre, et qui dépend de ressources dont il dispose, ressources matérielles bien sûr, mais aussi cognitives et culturelles. Le comportement social ne saurait donc simplement dépendre de l'obéissance mécanique à un système de normes: son explication impose de prendre en considération la position particulière de chaque membre de la population étudiée ${ }^{12}$. 
13 Il faut découvrir et mesurer, à partir de cas individuels, les différences de culture matérielle (la forme ou d'autres phénomènes physiques) et chercher ensuite les causes de ces différences, comme par exemple les facteurs socioculturels, puis déterminer les modèles d'habitat.

14 Il reste finalement à examiner, derrière les diverses formes et modèles d'habitat, les comportements, la mentalité ou les modes de vie, qui peuvent être aussi très différents.

15 Il serait indispensable d'employer, dans cette phase de l'analyse, d'autres types de sources, comme les récits de voyages, les procès-verbaux, les remarques des médecins, et des travaux d'ethnologues qui décrivent les gestes quotidiens de l'homme dans l'espace et aident à interpréter leur sens.

16 Il faut mentionner encore une dimension de la problématique de la recherche historique sur l'habitat. Les phénomènes de l'habitat ont beaucoup évolué au cours du temps et montrent de très grandes différences selon les époques. La transformation des formes joue donc un rôle d'indicateur des changements socioculturels.

17 Ce qui nous semble très important, c'est la capacité de l'organisation spatiale - donc de l'espace social et culturel -

[...] d'acquérir des propriétés nouvelles dont les caractères dépendent du passé et qui transforment les structures anciennes pour donner naissance à des fonctionnements neufs ${ }^{13}$.

Grâce aux traces et aux empreintes laissées par les individus, un détective peut découvrir la vérité. La tâche de l'historien est différente et plus compliquée. Il ne peut jamais retrouver vraiment la réalité socioculturelle de l'habitat puisqu'il n'entrera jamais dans les maisons et ne verra ni n'entendra jamais leurs habitants.

Bernard Lepetit entretenait avec la Hongrie des relations privilégiées. Outre les conférences qu'il a données à Budapest (à l'école doctorale de l'EHESS et à l'université Eötvös Loránd), quatre étudiants hongrois - Gábor Czoch, Peter Granasztói, Katalin Markalf et Gábor Sonkoly préparaient leur thèse avec lui. En référence à ses travaux et poür rendre hommage à sa mémoire, ils ont rédigé des articles sur l'histoire de la Hongrie.

\section{NOTES}

1. «Les représentations de la ville. Pour quoi faire?", in F. Walter (dir.), Vivre et imaginer la ville, XVIII ${ }^{e}$-XIX ${ }^{e}$ siècle s, Genève, 1988, p. 26.

2. Walter Benjamin, Paris, capitale du XIX ${ }^{e}$ siècle. Le livre des passages, Paris, Le Cerf, 1989, p. 239.

3. H.-E. Bödeker, «Marchands et habitat: le nord-ouest de l'Allemagne vers 1800 ", Revue d'histoire moderne et contemporaine, 1994, t. XLI, n ${ }^{\circ} 4$, p. 571-600; C. Bromberger, "L'habitat et l'habitation : des objets complexes. Quelles directions pour une analyse ? ", Habitat et espace dans le monde rural, Paris, MSH, 1988, p. 3-15; T. Hofer, «Le système des objets. Analyse ethnographique des équipements et ensembles d'objets ", Népi kultura-Népi tarsadalom, 1983, p. 39-65 ; Amos Rapoport, Pour une anthropologie de la maison, Paris, Dunod, 1972 ; A.-J. Schuurman, "Sozialgeschichte einer landlichen Wohnkultur in den Niederlanden im 19. Jahrhundert. Die Gegend von Zaanstreek bei Amsterdam um 1860 », in H.-J. Teuteberg (dir.), Homo habitans, 1985, 
p. 43-65 ; H.-J. Teuteberg, « Betrachtungen zu einer Geschichte des Wohnens », in H.-J. Teuteberg (dir.), Homo habitans, 1985, p. 1-23.

4. C. Bromberger, op. cit., p. 3.

5. H.-E. Bödeker, op. cit., p. 571.

6. W. Benjamin, op. cit., p. 239.

7. H.-E. Bödeker, op. cit., p. 573.

8. A. Rapoport, op. cit.

9. Le sujet de mes recherches : le changement de l'habitat dans un bourg de la Grande Plaine de Hongrie (1750-1850).

10. Une acre $=5754,642 \mathrm{~m}^{2}$.

11. K. K. Csilléry, "La différence de l'habitat selon les couches sociales », Ethnographia, 1985, p. 200-201.

12. P.-A. Rosental, « Construire le « macro » par le « micro »: Fredrik Barth et la microstoria », in J. Revel (dir.), Jeux d'échelles, Paris, EHESS, 1996, p. 145.

13. B. Lepetit, op. cit., p. 10.

\section{AUTEUR}

\section{PETER GRANASZTÓI}

Licencié en histoire et en ethnographie.

EHESS, sous la direction de Bernard Lepetit : Membre de l'Atelier franco-hongrois de sciences sociales, Budapest.

DEA « Histoire et civilisations », 1996.

Conservateur du musée d'Ethnographie, Budapest.Comme la flèche de Zénon, le commentaire n'atteint jamais son but et l'analyse, qu'elle soit celle des contemporains ou, a posteriori, celle des historiens ne rejoint jamais le réel. [...] L'opacité du réel n'est pas réductible et c'est banalité de dire que la valeur d'une analyse réside dans sa richesse. Le progrès du savoir historique passe ainsi par la complexification raisonnée des modèles qui rendent compte des processus passés. En inscrivant son travail dans une longue chaîne de commentaires, l'historien le sait bien : le mot fin ne sera jamais trouvé.

Bernard Lepetit 